\title{
Los jóvenes en favelas de Rio de Janeiro, Brasil: de la vulnerabilidad social a las oportunidades para el desarrollo humano
}

\author{
Youths in the shantytowns (favelas) of Rio de Janeiro/Brazil: \\ from social vulnerability to opportunities for human development
}

${ }^{1}$ Laboratório de Currículo e Ensino, Núcleo de Tecnologia Educacional para Saúde, Universidade Federal do Rio de Janeiro. Av. Carlos Chagas Filho, Cidade Universitária. 21941-590 Rio de Janeiro RJ Brasil.

gfigueiredo.ufrj@gmail.com

\begin{abstract}
Based on a review of living conditions in the complex and dynamic reality of the shantytowns ("favelas") of Rio de Janeiro and the main difficulties facing the human development of youths in this context, we analyze the social protection function involved in educational projects that offer new opportunities for life. In this article we analyze the relationship between the variables of social exclusion, poverty and violence, jointly grouped in the social vulnerability category, and the variables related to opportunities for human development grouped in the resilience category. The socio-educational projects constitute an important factor of resilience, able to influence the subjective development of young people and impact the improvement in the quality of life in the favelas. The social recognition and the relationship of trust established with educators and other youths in similar situations foster efforts to develop changes in attitude and to build new possibilities of life in spite of social vulnerability. The opportunity to experience interpersonal relationships, emotional bonds and positive social interaction can promote changes in the world view of youths and elicit a desire to change their living conditions and enhance their projects for the future.

Key words Youth, Vulnerability, Resiliency, Education, Human development
\end{abstract}

Resumen Desde una reflexión acerca de las condiciones de vida en la realidad compleja y dinámica de las favelas de Rio de Janeiro y, de las principales dificultades generadas para el desarrollo humano de la juventud en este contexto, analizamos la función de protección social que asumen los proyectos educativos al proporcionar nuevas oportunidades de vida. En este articulo analizamos la relación entre las variables exclusión social, pobreza y violencia, integradas en la categoría vulnerabilidad social y las variables relacionadas a las oportunidades para el desarrollo humano integradas en la categoría resiliéncia. Los proyectos socioeducativos se constituyen en importante factor de resiliéncia capaz de influir en el desarrollo subjetivo de los jóvenes e impactar en la mejora de la calidad de vida en la favela. El reconocimiento social y el vinculo de confianza establecido con educadores y otros jóvenes en situación similar estimula el esfuerzo por desarrollar cambios de actitud y la construcción de nuevas posibilidades de vida a despecho de la vulnerabilidad. La oportunidad de vivenciar relaciones interpersonales, vinculos afectivos y lazos sociales positivos promueve cambios en la visión de mundo de los jóvenes y despierta el deseo de cambiar sus condiciones de vida y proyectos de futuro.

Palabras clave Juventud, Vulnerabilidad, Resiliéncia, Educación, Desarrollo humano 


\section{Introducción}

El tema del desarrollo de la juventud en las favelas no es un fenómeno exclusivo de una ciudad o de un país. Por lo tanto, habrá que tener muy presente las coordenadas espacio temporales de una temática tan amplia y variada. Por otro lado, el mismo término arrastra un paquete de cuestiones transversales no solo difíciles de tratar con las herramientas conceptuales disponibles, sino también vergonzantes porque hieren la dignidad humana. Así hablar de favelas puede significar abordar, entre otros temas, cuestiones referidas a: la violencia, la exclusión social, la pobreza, el crimen organizado, el comercio de drogas, la ocupación del suelo y la vivienda precaria, la corrupción policial, la presencia desdibujada de las instituciones del Estado o del adormecimiento de las prácticas socioeducativas.

En el estudio de los fenómeno que se pasan en las favelas generalmente se tiende a simplificar y falsear la realidad cuando las favelas son caracterizadas solamente como comunidades pobres, excluidas, situadas en los espacios urbanos que carecen de infraestructura de saneamiento básico y urbanización que viven en contextos de violencia. Aunque cada una de estas condiciones, y en su conjunto, han estado presentes y pueden seguir estándolo en las favelas actuales, buscamos recolocar estas condiciones teniendo en cuenta otras realidades silenciadas.

Aunque todos estos temas forman parte de la imagen más común ampliamente compartida sobre la realidad de las favelas conviene no perder de vista que tal representación se ajusta solo parcialmente y que, históricamente, también existen innúmeros factores de protección social que promueven la resiliéncia y ofrecen nuevas oportunidades para el desarrollo de los jóvenes. Justamente es esta condición ambigua del contexto social en que viven los jóvenes de las favelas que se pretende analizar en este artículo.

\section{Favela: la definición de un concepto dinámico y complejo}

La complejidad y diversidad de las múltiples realidades posibles cuando se habla de las favelas está relacionada con la etapa de desarrollo histórico en que se encuentran estos agrupamientos humanos. De forma general, cuanto más tiempo existen desde su fundación, mejores serán las condiciones de vida. Las variaciones surgen aún de la capacidad de organización de cada comuni- dad, de la fuerza de sus asociaciones, agremiaciones e instituciones. O sea, depende de la voluntad colectiva y de la capacidad de lucha, sea para defenderse, para lograr conquistas políticas, o para mejorar las condiciones materiales de vida.

A pesar de los contextos sociales, históricos y económicos distintos en cada ciudad, el caso de las favelas de Rio de Janeiro puede considerar-se típico de las grandes ciudades en Latinoamérica y el presente artículo también podrá contribuir para la reflexión de la problemática desde una perspectiva social, histórica y critica. La construcción colectiva de conocimiento teórico y alternativas practicas a los problemas generados por el crecimiento urbano desigual puede evitar sus consecuencias perversas al desarrollo humano también en otras partes del mondo. Aunque las diferencias regionales sean importantes $y$, en alguna medida, el fenómeno reciba nombres locales (favela, slums, chabolas, pueblos jóvenes o katchi obadi), las raíces de los procesos sociales de explotación del trabajador e injusticia social que les configuran, tienen aspectos similares.

Oficialmente, para el Instituto Brasileño de Geografía y Estadística ${ }^{1}$, favela es: un conjunto constituido de, por lo menos, 51 unidades habitacionales ocupando, o que haya ocupado hasta un período reciente terreno o propiedad alheña (pública o privada), dispuesto, en general, de forma desordenada y densa, en su mayoría, carentes de los servicios público esenciales. La reciente substitución del término "favela" por el término "comunidad" en la política brasileña no parece haber contribuido a la comprensión de la dinámica de los procesos sociales y a la des-estigmatización de los territorios ${ }^{2,3}$.

El concepto de "favela" es un importante recurso político para que estas comunidades puedan exigir inversiones públicas y condiciones adecuadas para el desarrollo humano y para la mejora de la calidad de vida en los territorios en que viven. Si por un lado, reafirmar el nombre, la cultura y la identidad de favela acentúa las diferencias dentro de la ciudad y crea tensión, por otro lado, el referente favela estimula la lucha por los derechos, favorece la formación de nuevas asociaciones, unifica la cultura local, caracteriza la identidad propia de la gente que ahí vive y activa el desarrollo político de estas comunidades ${ }^{2,3}$.

Es relevante considerar aún que esta generación de jóvenes que vive en las favelas de Rio de janeiro es más educada que nunca, tienen acceso - aún que de forma escasa - a la cultura, a los medios de comunicación, a la informática, y son fruto del trabajo casi invisible de innumerables 
individuos, grupos, colectivos, asociaciones, organizaciones, fundaciones y empresas que vienen desarrollando en las últimas décadas un importante trabajo de concienciación política, de desarrollo de capacidades humanas, de formación, de organización.

La población de las favelas sabe que sus condiciones de vida y que sus posibilidades y oportunidades de futuro son muy diferentes de la gente de la clase media y alta con quien conviven diariamente en el ir y venir de la ciudad. Muchos se rebelan, se encuentran con/en la marginalidad, algunos están agresivos, otros optan por caminos arriesgados, y muchos se esfuerzan por cambiar la realidad de vida (individual, familiar y colectivo-social), a cada dia.

Por otro lado, a cada día, la prensa sigue divulgando los conflictos en las favelas. Cada día, son asesinados más jóvenes, cada semana mueren decenas de inocentes, cada mes la gente de la favela sufre con invasiones de grupos rivales, pandilhas armadas, milicias politicas y con la violenta intervención policial. El problema gana, cada día, dimensiones de mayor impacto en la opinión pública y verdaderamente se constituye como el principal problema a ser afrontado por la sociedad brasileña en rumbo a su desarrollo.

Cuanto al método, cabe considerar que este estudio se basa en nuestra investigación de doctorado ${ }^{2}$, con trabajo de campo integrado, quali/ quanti y metodológicamente estructurado, llevado a cabo entre los años de 2006 y 2011 gracias a la beca para investigación aportada por la Agencia Española de Cooperación Internacional y Desarrollo (AECID). En este artículo, los datos se refieren principalmente a cuatro favelas en la ciudad de Rio de Janeiro, a saber: Favela de Curicica (Jacarepagua), Favela de Manguinhos (Bonsucesso), Favela da Maré (Vila do João) e Favela do Mendanha (Campo Grande).

\section{El dinámico contexto de vulnerabilidad y protección social}

El slogan adoptado por el gobierno de izquierda coordinado por el Partido de los Trabajadores que eligió el Presidente Luis Ignacio Lula da Silva como Presidente del país en 2002 revela el principal desafío al desarrollo humano que se afronta: poder ejercer el derecho a la relación participativa ciudadana en la sociedad del bienestar. "Brasil: Un país de todos" es la forma más clara de dejar constancia de las dificultades, obstáculos y retos que supone garantizar el derecho a la subsisten- cia y la calidad de vida a distintos colectivos. Sin embargo, una de las características más utilizadas para referirse al país es su desigualdad social. Los contrastes son muy evidentes y fragmentan las ciudades y las Unidades de la Federación (estados) hasta el punto de decirse que hay características de países pobres y ricos conviviendo codo con codo en un mismo país. La pobreza y la exclusión social en que viven amplias camadas de la población contrasta con el estilo de vida de las clases medias y altas creando situaciones sociales bastante conflictivas, que son agravadas por la creciente violencia generada por la actuación de las mafias.

Recientemente Brasil ha ganado cada vez más expresión en el escenario internacional y surge como una fuerte potencia mundial formando junto con Rusia, India y China el grupo del BRIC, países en vías de desarrollo que más han crecido en los últimos años. En 2006, la esperanza de vida al nacer era de 72 años, la tasa bruta de mortalidad del 6\% al año; la tasa bruta de natalidad de $18 \%$ al año y la tasa de crecimiento anual de la población fue de 1,73\%. En el ano de 2006 más de $40 \%$ de la población económicamente activa trabajaba en condiciones informales. Sin embargo, en el año de 2008, casi $30 \%$ de los brasileños aún estaban en situación de pobreza y cerca de $20 \%$ de la población urbana vive en favelas. En 2012 el Gobierno Federal adopta el slogan: País rico é país sem pobreza, revelando que el principal desafío al desarrollo en el país es distribuir la riqueza generada, disminuyendo así la desigualdad entre clases sociales y revertiendo el histórico proceso de exclusión social que caracteriza su sociedad $^{1}$.

Brasil aún presenta contradicciones sociales incompatibles con el status de desarrollo humano esperado de los países considerados "desarrollados". Ya es sabido que el principal problema ahí, no es la pobreza, sino, la distribución de la riqueza. Basta visitar cualquier ciudad brasileña para percibir la gravedad de las diferencias. Los pueblos, los barrios, las ciudades, las provincias, las macro regiones, todos los espacios están separados entre los ricos y los pobres. Muchas veces tendemos a decir que son dos países, dos realidades, dos culturas, dos universos, sin embargo, el conflicto reside en el hecho de que la realidad del país es única y que en ella conviven codo a codo todos los sectores sociales, que se encuentran, se enfrentan y disputan, lo que resulta en una sociedad fuertemente fragmentada. Así, percibimos que, por un lado, la exclusión es el arma que se utiliza para hacer prevalecer el poder de los que 
secuestran la riqueza, y, por otro, las terribles consecuencias de la violencia es generada por la privación del derecho a una vida digna.

La ocupación del espacio urbano en Brasil es reflejo de las contradicciones y luchas internas de la propia sociedad. Las favelas son un fenómeno social que fue se constituyendo históricamente como una cuestión relevante para la sociedad brasileña y el proceso político- económico influye en la conformación de estos espacios. Son inúmeros los problemas, los desafíos, las resistencias, los movimientos populares, las ideologías, la fuerza, la disputa de poder y el conflicto generado por la definición del término "favela" y sufre influencia de los distintos modelos de urbanización de la ciudad que consideran la favela algunas veces como causa y otras como consecuencia de los problemas de las grandes ciudades brasileñas.

La pérdida de solidaridad orgánica o cohesión social descubre que el hundimiento de la vida de las personas y los grupos tiene que ver con el bloqueo y/o descomposición de las relaciones participativas de naturaleza afiliativa y económica². En esta sección, se revisa la forma de relacionar los conceptos tradicionales de pobreza, exclusión y violencia con la nueva realidad diversa de las favelas de Río de Janeiro. El concepto de vulnerabilidad social nos va ayudar a situar las condiciones de vida de los jóvenes de hoy en un espacio susceptible de ser gestionado de forma positiva para el desenvolvimiento y enriquecimiento de sus vidas, pero que aún sólo puede ser percibido como un escenario más estresante que incitador de cambios positivos por la sociedad.

\section{La pobreza como carencia}

De acuerdo con Perona y Rocchi ${ }^{4}$, el concepto de pobreza está vinculado al estudio de la problemática que conduce a la marginalidad social con todo lo que ello conlleva de ruptura de lazos sociales y destrucción de la vida de las personas. La investigación sobre la pobreza ha posibilitado distintos planteamientos como los centrados en las necesidades básicas insatisfechas que tiene que ver con la falta de ingresos o el adoptado por las Naciones Unidas sobre el concepto de carencia de capacidades, elaborado por el economista Amartya $\mathrm{Sen}^{5}$, y que considera como determinante del concepto la falta de recursos, habilidades y libertades o el enfoque de la privación (deprivation) que contempla el problema de no poder satisfacer las necesidades básicas pero también considera la situación de la pobreza relativa, es decir, cuando en la canasta de bienes y servicios que pueden asegurar una vida digna a un individuo o a una familia no puede incluir las nuevas necesidades de acuerdo a las convenciones y estándares de una sociedad determinada.

En el artículo que estamos revisando ${ }^{5}$ se destaca que los enfoques carenciales de la pobreza ponen en evidencia la ausencia del ejercicio pleno de los derechos que competen a las personas. El colectivo sumergido hoy en la pobreza carencial tiende a perpetuarse porque sus condiciones de vida afectan al crecimiento personal y social de los jóvenes. La situación de carencia y deterioro afecta a las nuevas generaciones al ocasionar la falta de formación en competencias actualizadas, el debilitamiento de las relaciones afiliativas y el desencanto sobre la influencia social y los asuntos públicos. Desde de esta mirada, se ha acuñado el término de nueva pobreza ${ }^{6}$.

De lo anterior se desprende que el problema de la pobreza es multidimensional y se debe atacar, como dice Martínez ${ }^{7}$ desde diferentes ángulos. Por un lado, se deben llevar a cabo políticas top-down (de arriba hacia abajo) que propicien la estabilidad y el crecimiento económico. Y, por otro, es imprescindible implementar políticas $b o$ ttom-up que promuevan las competencias de las personas, la forma de obtener ingresos y la implicación social en la búsqueda de soluciones para mejorar las condiciones de vida.

\section{De la pobreza como carencia a la exclusión social}

La pérdida de participación e integración en la vida social es fenómeno ligado a la desventaja social pero que no se limita a las condiciones de la pobreza económica. De acuerdo con Comisión Europea ${ }^{8}$ y Alkire ${ }^{9}$ la situación de inclusión o exclusión social de un individuo se define, por tanto, en términos relativos a la situación del individuo con respecto a la población considerada mayoritaria, valorando la situación de la persona con respecto a la posibilidad de ejercer los derechos sociales de la mayoría.

Al mismo tiempo que se habla de la profundidad del concepto, que se presenta como un término abarcador de toda clase de privaciones ${ }^{6}$ cabe señalar, por un lado, la dificultad que entraña fijar su especificidad conceptual y, por otro, el largo recorrido que éste ha seguido desde su nacimiento en Francia ${ }^{10}$, pasando por su penetración en los estudios sociales de los países más desarrollados, con excepción de USA (los estu- 
diosos norteamericanos están más inclinados a utilizar el término underclass), hasta ser aceptado como categoría analítica de la realidad social de los países latinoamericanos ${ }^{11}$.

Con palabras de Delors (según Abrahamson $)^{12}$ pronunciadas en la conferencia de clausura Seminario Luchar contra la Exclusión Social ${ }^{13}$, "... en el futuro continuaremos distinguiendo entre pobreza y exclusión social [...] aunque exclusión incluye pobreza, pobreza no incluye exclusión" y remarcaba que la exclusión no es un fenómeno marginal sino un fenómeno social que cuestiona y amenaza los valores de la sociedad.

El análisis de Atkinson ${ }^{14}$ sobre la exclusión social, concebido des una visión jerárquica de la sociedad formada por diferentes grupos que se defiende unos de otros al tiempo que promueven solidaridad dentro del propio grupo, se centra en tres dimensiones: en primer lugar, la línea de la exclusión surge cuando se tienen en cuenta las condiciones de vida de los diferentes grupos, comunidades, familias e individuos que conforman un sociedad y se observa la presencia de claras e importantes desventajas de unos en relación con el resto. No se puede hablar de exclusión y de inclusión sin tener en cuenta la realidad social de un determinado territorio en su momento histórico. Junto con la relatividad del fenómeno, debe considerarse, en segundo lugar, el papel que juegan las personas con su comportamiento. La capacidad de obrar (agency) de la gente en términos de autoexclusión o elecciones, y de excluir a los demás. Por último, señala transmisión generacional de las condiciones de vida que hacen posible la exclusión.

Al hilo de estas consideraciones que señalan la relatividad del concepto, Perona y Rocchi ${ }^{4}$ citan textualmente la explicación que da Atkinson: [la exclusión] "constituye la contrapartida de la inclusión, es decir se está excluido de algo cuya posesión implica un sentido de inclusión. No es un concepto dicotómico que divide a los individuos o grupos en dos; existe una serie de situaciones intermedias entre ambos estados" [...] "Es también relativo porque varía espacial e históricamente, en los contextos situados; por otra parte tiene mayor potencialidad analítica para referirlo a aquellas situaciones que implican "fuerte acumulación de desventajas".

\section{El lugar de la violencia}

El impacto de la violencia en la vida de los jóvenes de las favelas es terrible. Cada año, cerca de 20 mil jóvenes en todo Brasil entre 15 y 24 años son víctimas de homicidio exclusivamente por causas relacionadas con la violencia, lo que representa más de $40 \%$ de las causas de muerte a esta edad. Además de los asesinatos, los jóvenes sufren diversas otras consecuencias de la violencia como el abandono, malos tratos, agresiones, abusos físicos y trastornos psicológicos ${ }^{15}$.

La prensa sigue divulgando los conflictos en las favelas. Cada día son asesinados más jóvenes, cada semana mueren decenas de inocentes, cada mes la gente de la favela sufre con invasiones de grupos rivales y con la violenta intervención policial. El problema gana cada día dimensiones de mayor impacto en la opinión pública y se constituye verdaderamente como el principal problema a ser enfrentado por la sociedad brasileña en rumbo a su desarrollo.

La concepción de violencia defendida por Wright Mills, Max Weber y Mao Tse Tung como manifestación del poder, comprendido como el dominio del hombre sobre el hombre y que exige la efectividad del comando fue reformulada por Arendt ${ }^{16}$. Para la autora, poder y violencia son temas opuestos. La violencia surge justamente cuando se debilita el poder instituido. Para ella, el poder, inherente a cualquiera comunidad política, resulta de la capacidad humana para actuar en conjunto, lo que requiere el consenso de muchos en cuanto al curso común de la acción. La violencia, por otro lado, surge de la desintegración del poder, cuando autoridades políticas ya no son respetadas de forma general.

Aún para Arendt $^{16}$ la violencia destruye el poder, no lo crea. La violencia y su glorificación se explica por la severa frustración de la facultad de actuar en el mundo contemporáneo, que tiene sus raíces en la burocratización de la vida pública, en la vulnerabilidad de los grandes sistemas y en la monopolización del poder, que secan las fuentes creativas. Para ella, el deterioro del poder por la carencia de la capacidad de actuar de forma concertada, en conjunto, es un convite a la violencia.

En un análisis del fenómeno de la violencia por medio de los problemas culturales estructurales y seculares en Brasil, Minayo ${ }^{17}$ afirma que las inmensas desigualdades de la sociedad brasileña se acumularon y no fueron superadas, sino contrariamente, crecieron con los cambios promovidos por la industrialización y la globalización. La presencia del tema violencia y criminalidad en la agenda de la ciudadanía de la sociedad sólo surgió en la década de los 80’s con el fin de la dictadura militar en Brasil, cuando los movimien- 
tos sociales para la democracia, instituciones de derecho, la fuerte presión de organizaciones no-gubernamentales y agencias humanitarias internacionales tornaron la violencia como una cuestión pública a ser debatida y enfrentada por la sociedad brasileña en su proceso de desarrollo. La autora afirma aún que las formas de violencia presentes en los últimos 25 años en Brasil son subproductos de la dimensión política, del poder, y denuncia la descomposición de los sistemas sociales promovidos por la dictadura militar.

El lugar de la violencia hoy también se encuentra en la manera de concebir la lucha contra la violencia que está en la calle. Turchetti ${ }^{18}$ ha realizado una interesante investigación sobre como la autoridad pública lucha contra las actividades criminales en la favelas de Río de Janeiro. Dichas actividades se despliegan por derroteros muy diversos. Desde la participación en el ilegal tráfico de drogas, la lucha interna entre comandos, la utilización de medios delictivos más comunes para sobrevivir, hasta la inclusión de las acciones de determinados cuerpos de policía. Turchetti ${ }^{18}$ al referirse al comportamiento de policía escribe: "En particular, la relación de la policía con los traficantes de drogas en Río de Janeiro es doble. Paradójicamente, se pelean entre sí por la capacidad de imponer sus reglas (las normas estatales o de las reglas Comando) sobre los territorios de las favelas; pero también son, en algunos casos, socios en un trato ilegal (obviamente) que ayuda a mantener el tráfico en la ciudad".

Por otro lado, la investigación que revisamos $^{18}$ identifica el modus operandi del llamado Batalhão de Operações Policiais Especiais especializado en la lucha contra el narcotráfico. Como su escudo indica, "un cráneo empalado con un cuchillo y dos pistolas", se trata de un cuerpo preparado para "dar miedo" e intimidar a los que viven en las favelas. Las acciones de limpieza conviven con la represión encargada de mantener el orden y no se trata de casos puntuales y aislados, es una política que se excede en sus competencias hasta tal punto que los derechos humanos no son respetados.

Es cierto, y así lo consigna la investigación ${ }^{18}$, que la propuesta de la Unidade de Policia Pacificadora tiene como cometido promover el bien estar de las personas que viven en la favelas. Esta debería ser la policía que se integra en el barrio, trabaja por su pacificación, regulariza y proporciona los bienes públicos de un barrio normal. Sin embargo, la dualidad vuelve a aparecer y son frecuentes las denuncias de abuso de poder, agresiones y violencia policial hasta al punto de se prohibir que la gente de las favelas saiga de casa después de las 22 horas. Parafraseando Freire, los oprimidos se convierten en opresores: O Irônico é que são eles mesmos pessoas oprimidas que se utilizam do poder para se tornarem os novos opressores $^{19}$.

La violencia se constituye actualmente en uno de los más consensuales problemas del país. La acción de las organizaciones criminales difundidas principalmente en las periferias y favelas de las grandes ciudades representan un fuerte riesgo para la democracia y para el efectivo ejercicio de la ciudadanía por parte de la población. La desigualdad social y la pésima distribución de la riqueza pasa factura, incrementa la violencia y representa una fuerte amenaza para el desarrollo humano en Brasil.

Por otro lado, existe una reconocida relación entre el desarrollo humano y la construcción de un ambiente de paz. Como afirma Morin ${ }^{20}$, en la paz el espíritu humano se abre al mundo. La apertura al mundo se revela por la curiosidad, por el cuestionamiento, por la exploración, por la investigación, por la pasión de conocer. Se manifiesta por la estética, por la emoción, por la sensibilidad, por el encantamiento frente al nacer y del poner del sol, de la luna, de las nubes, de los abismos, del canto de los pájaros, y esas emociones vivas estimularán el cantar, dibujar y pintar. Incita a todos los inicios. El espíritu humano se sentirá entonces estimulado por subida en el mundo. Justamente por eso optamos por trabajar con el arte para estimular la interacción social y el desarrollo humano de estos jóvenes.

Sin embargo, la idea y la experiencia de seguridad humana, y de paz, deben ser también construidas a otros distintos niveles, siendo prioritario el papel que juegan las instituciones, y la política, para que estrategias y metodologías, se tornen efectivamente instrumento de trabajo para la construcción de la paz, la convivencia ciudadana y la posibilidad de construir e imaginar nuevas perspectivas de vida y proyectos para el futuro. Sin embargo, esto solamente se hace viable y posible con la reducción de la inmensa desigualdad que caracteriza la sociedad del país.

\section{La situación de vulnerabilidad social}

Vulnerabilidad social es un concepto utilizado por algunos autores y estudiosos para describir una zona intermedia entre la integración social y la marginalidad-pobreza ${ }^{21}$. Una persona vulnerable es aquella cuyo entorno personal, fami- 
liar-relacional, socio-económico o político-administrativo padece alguna debilidad y, en consecuencia, se encuentra en una situación de riesgo que podría desencadenar un proceso de la exclusión social. El nivel de riesgo será mayor o menor dependiendo del grado de deterioro del entorno. Dowdney ${ }^{22}$ afirma que los niños buscan el trabajo en las mafias como única opción generada por la falta de otras oportunidades.

La noción de vulnerabilidad social ayuda a identificar a grupos sociales e individuos, que por su menor disponibilidad de activos materiales y no materiales, quedan expuestos a sufrir alteraciones bruscas y significativas en sus niveles de vida, ante cambios en la situación laboral de sus miembros activos. La introducción de la categoría conceptual "condiciones de vida" se vincula a la necesidad de abarcar los diversos planos y dimensiones tanto de la vida privada como comunitaria. Las "condiciones de vida" aluden al equipamiento y/o provisión de bienes del hogar en relación a la cantidad de miembros, a las características de la inserción ocupacional de los miembros, a los niveles de educación alcanzados, al acceso a los beneficios sociales y a la posibilidad de expresión y participación en la vida pública.

A partir de la experiencia de educadores sociales en diferentes favelas de Rio de Janeiro, Figueiredo \& Wimmer ${ }^{23}$ y Figueiredo ${ }^{2,24}$ describen los principales problemas que caracterizan el contexto de vulnerabilidad social en que viven los jóvenes de las favelas en Rio de Janeiro. De acuerdo con los autores, los principales problemas que crean condiciones de riesgo en el entorno del desarrollo de los jóvenes son: i) Alta tasa de desempleo, gran número de trabajadores sin papeles de la seguridad social y numerosas personas trabajando en el mercado informal o desocupadas. El bajo valor de los sueldos y la gran cantidad de gente que recibe un sueldo inferior al salario mínimo también es un grave problema; ii) Precariedad de las instalaciones, falta de profesionales e insuficiencia de plazas en las escuelas públicas de nivel fundamental y en los Institutos. Muchos jóvenes con menos de 15 años trabajan durante el día y estudian por la noche y muy pocos estudian en los Institutos durante el día. Cerca de 50\% de los jóvenes que concluyen la enseñanza fundamental ni mismo consiguen hacer la matricula en los institutos de secundaria por falta de plazas disponibles. Así, abandonan los estudios; iii) Dificultad de acceso al sistema público de salud y baja calidad de la atención; iv) Insuficiencia y precariedad de guarderías de niños y jardines de infancia lo que imposibilita a muchas mujeres de procurar un empleo. Este problema puede ser evidenciado por el gran número de cuidadoras autónomas de niños y guarderías ilegales que hay en las favelas; v) El embarazo en la adolescencia es muy común. El alto número de casos de embarazo de las adolescentes denuncia revela que los jóvenes viven el embarazo precoz como una realidad común. $\mathrm{Mu}$ chas optan por realizar el aborto (práctica ilegal en Brasil) y para esto consumen medicamentos industrializados. Algunas pocas buscan realizar el procedimiento en clínicas ilegales debido al alto valor cobrado por el procedimiento en el mercado privado y la inexistencia de la opción en la salud pública; vi) Es común la inexistencia de un sistema de alcantarillado y cañería para los residuos fisiológicos provenientes de las viviendas y que estos residuos fisiológicos se acumulen por las calles de tierra, o que sean diseccionados al río más cercano sin cualquier tratamiento anterior. El río contaminado recibe el nombre de "valão" y recibe, además de los residuos fisiológicos, la basura proveniente de las viviendas. Generalmente el valão no está cubierto y atraviesa gran parte da favela. La contaminación disemina vectores de enfermedades como ratones, cucarachas y mosquitos; vii) La basura está tirada por las calles y se acumula en algunas zonas de la favela. En la realidad, los vecinos sacan la basura de casa y la tiran para el lado de fuera de las viviendas, sin embargo, como las viviendas están construidas de forma desordenada, la basura se acumula y se distribuye por las calles con la ayuda de perros, gatos y de los ratones; viii) Otro problema importante es la presencia de mafias y organizaciones criminales con grande número de personas utilizando armas de fuego, el comercio de drogas ilegales, conflictos armados frecuentes con grupos rivales o con la policía. Abuso del poder, de la fuerza y practica de tortura como presencia frecuente de la violencia de la policía. Sensación de inseguridad y de vivir en estado de guerra cotidiana con el ruido proveniente de los disparos de las armas; ix) Alta incidencia de problemas relacionados con la nutrición (principalmente obesidad y desnutrición crónica) debido a tres niveles de deficiencia en la alimentación: En el primer nivel hay acceso restricto a los géneros alimenticios más caros (principalmente proteínas como la carne, leche, quesos, yogures), así, hay una opción por alimentos más baratos como los carbohidratos, harinas y azúcares en general. En el segundo nivel, hay los casos de acceso ocasional e irregular a los alimentos que se evidencia por el alto índice de subnutrición y se revela en el drama del hambre crónica. $\mathrm{x}$ ) Hay un número grande de personas con disturbios psicológicos y emocionales 
provocados por las precarias condiciones de vida. Son manifestaciones muy comunes la depresión, la agresividad, la frustración, el miedo, la sensación de incapacidad y la inestabilidad emocional. La mayor parte de esta gente está sin amparo institucional de los servicios de salud y el consumo de fármacos psicotrópicos, de alcohol y de drogas ilícitas denuncian esta realidad. xi) Precariedad de las condiciones de vivienda. La mayor parte de las casas está construida de ladrillos y cemento, sin embargo, son casi siempre muy pequeñas y tienen solamente una habitación, un baño y la cocina. El ambiente interno de la vivienda es generalmente oscuro y con bastante humedad debido a la ausencia de ventanas y a la precariedad de la construcción de las paredes y del tejado. Casi todas las casas poseen, aunque de forma ilegal, energía eléctrica y un número más pequeño tiene acceso al agua tratada proveniente del sistema de abastecimiento público; xii) Alta prevalencia de enfermedades infecto-contagiosas, infecto-parasitarias e infecto-virales. Son frecuentes los casos de enfermedades respiratorias, dermatológicas y crónicas, donde se destacan la hipertensión, la diabetes. Enfermedades como la tuberculosis, la lepra y enfermedades sexuales también son muy frecuentes; viii) Precarias condiciones de higiene causadas, no solamente por el acceso precario a los productos de limpieza e higiene personal, como también por la falta de información, por la gravedad de los problemas sociales, por la desesperanza, por la baja autoestima personal, por la depresión crónica y por las limitadas perspectivas de cambio de vida. ix) La violencia familiar se manifiesta principalmente por la violencia de género con grande número de agresiones a las mujeres, $y$ también es frecuente la violencia; $\mathrm{x}$ ) la negligencia y el abandono de niños y jóvenes. La prostitución de adultos jóvenes y adolescentes, principalmente del sexo femenino también se constituye en un problema ya que la práctica se disemina por la ausencia de opción y de ocupaciones que puedan generar algún dinero.

Por fin, como bien resume Figueiredo ${ }^{24}$, cuando se solicita a los moradores de las favelas para identificar y priorizar de forma resumida los principales problemas que dificultan la vida en las favelas, las prioridades casi siempre se repiten en esta orden: Condiciones de las viviendas; Hambre o incapacidad de alimentar la familia de forma adecuada; Desempleo y ausencia de dinero (mismo un sueldo informal); Precariedad del sistema de cañería y acumulación de basura por la calle; Violencia provocada por organizaciones criminales y por la policía.
La preocupante enumeración de problemas que afectan a las favelas como espacio socioecómico, sociopolítico, socioeduativo y sociourbano (Perona y Rocchi ${ }^{4}$; Turcheti ${ }^{18}$ ) convive con los planes de intervención, que impulsa el Estado, basados en criterios de intervención territorial sistémicos de carácter interagencial y multidisciplinarios, con énfasis en la legalidad de las acciones, gradualidad en las respuestas y marcado interés en las acciones de prevención.

Los proyectos de intervención social se constituyen en los más importantes factores de protección social ${ }^{23}$. Junto con las familias, los grupos espirituales, las escuelas y la comunidad local, suelen ser los pocos recursos de resiliéncia que garanten oportunidades para el desarrollo del talento de los jóvenes. Pero hay también los jóvenes que ya están en el mercado de trabajo (formal o no), ejerciendo actividades (legales o no), para vivir la vida como la vida é.

\section{Algunas relaciones entre los conceptos de pobreza, violencia y exclusión social}

Con el objetivo de mejor analizar los factores de vulnerabilidad y de protección social a que están expuestos los jóvenes en favelas, seguimos Figueiredo $^{2}$ cuando afirma que pudo verificar que este territorio (favela) está fuertemente determinado por los factores de vulnerabilidad inherentes a la situación de pobreza, violencia y exclusión social que conforman la realidad concreta social, cultural y históricamente ubicado. Al discutir los factores determinantes de la exclusión social de los jóvenes involucrados en el estudio, la ausencia de la formación profesional, la precariedad de la educación formal, el analfabetismo básico, la ausencia de infraestructura de cultura y ocio, la debilidad del sistema de atención a la salud, el limitado acceso a la tecnología y a la prensa de calidad y por último factores que limitan la movilidad y el transporte de los jóvenes por la ciudad son los que aún determinan y limitan el desarrollo de los jóvenes en las favelas. Estos factores demuestran que la exclusión social es uno de los elementos más fuertemente vinculados a la situación de vulnerabilidad en que se encuentran los jóvenes atendidos por los proyectos de intervención estudiados ${ }^{2}$.

$\mathrm{Al}$ analizar los factores relacionados a la pobreza, el autor destaca el desempleo/desocupación de los jóvenes como el factor más prevalente encontrado en nuestra investigación, seguido por los factores psicológicos negativos como depresión 
$y$ ansiedad que se vinculan directamente a la realidad de vida en que se encuentran los jóvenes atendidos por las instituciones que participaron del estudio. Otros factores relacionados a la pobreza que deben ser destacados son la desestructuración familiar y la precariedad de la vivienda. Por fin, al analizar los factores de vulnerabilidad social relacionado al contexto de violencia, son muy frecuentes la represión policial, la presencia frecuente de homicidios y asesinatos de la población de las favelas y un contexto marcado por agresiones psicológicas relacionadas al prejuicio y la discriminación de los jóvenes².

Estos resultados están de acuerdo con los resultados revelados por el estudio de Arpini $^{3}$ cuando concluye que los jóvenes que viven en contextos de exclusión social están frecuentemente expuestos a otros tipos de violencias físicas como puniciones, agresiones y violaciones además de diversos tipos de violencias psíquicas con privaciones de orden afectivo, acosos y diversos tipos de descalificación que viven en sus relaciones sociales.

Calificando la mirada acerca del fenómeno de la violencia, Minayo ${ }^{17}$, afirma que el crecimiento de la violencia en Brasil es reflejo de la exacerbación de nuevas formas de sociabilidad, construidas con ingredientes que hacen parte de una emergente ideología dominante: la que mezcla el consumismo con el hedonismo y el individualismo con el egocentrismo y el inmediatismo. Son en estas condiciones y circunstancias de escasez creciente de oportunidades y perspectivas que se construyen las tasas de criminalidad y demás consecuencias de la violencia en el país.

Como se puede constatar, la vida en las favelas está fuertemente determinada por los contextos de exclusión y violencia que se experimenta en el cotidiano de vida de la gente, conformando su subjetividad y creando racionamientos que pueden muchas veces parecer absurdos e incoherentes. Frecuentemente los jóvenes reclaman la necesidad de intercambiar ideas, de conversar, de construir colectivamente otras formas de vida y su carencia afectiva y poca madurez emocional delante de temáticas tan complejas como la muerte, la violencia, la exclusión y la planificación de su futuro personal. Solamente al comprender la cultura en que están inseridos estos jóvenes y las pocas posibilidades que perciben cuanto al futuro es que podemos comprender el modo como perciben su existencia y articulan sus pensamientos. La proximidad cotidiana con la mafia, la revuelta con las condiciones de vida y el miedo están casi siempre presente en sus discursos, sea legitimando la acción del trafico de drogas como trabajo e inserción en el universo productivo, sea justificando su fuente de renta. Asimismo, es común la experiencia de la muerte, el conflicto ético delante de experiencias violentas y el sufrimiento causado por tales condiciones de vida ${ }^{24}$. Al hablar del hambre, del embarazo precoz, de la violencia de la policía y de la falta de oportunidades concretas en las favelas, estos jóvenes denuncian la precariedad de las acciones públicas en el enfrentamiento del problema y comunican su angustia frente a su situación concreta de vida ${ }^{2,23,24}$.

Por fin, haciendo el contrapunto de la vulnerabilidad social, están también presentes algunos factores resiliéncia y protección. Se puede observar la importancia de la familia, del grupo de amigos, de la escuela y de los educadores sociales en el desarrollo de sus percepciones sobre la vida, sus pensamientos cuanto al futuro y la necesidad que expresan de una interacción social más positiva $^{2,24}$.

\section{Factores de resiliéncia y protección social}

En importante artículo de revisión sobre el tema de la resiliéncia, Olsson et al. ${ }^{25}$ presentan los datos de la investigación de Fergusson \& Lynskey que comprobaron, por medio de un estudio longitudinal con jóvenes, la relación lineal y directa entre el número de factores de riesgo en el medio ambiente del individuo con el número de problemas psicosociales que estos jóvenes presentaron. Sin embargo, afirman que así como los factores de riesgo pueden aumentar el número de problemas psicosociales, el efecto de múltiples factores de protección también puede ampliar la resiliéncia de los jóvenes por medio de una cadena positiva de condiciones que influyen de forma positiva en su desarrollo personal. Los autores definen la resiliéncia desde una perspectiva multifactorial y la definen como un proceso dinámico de adaptación al riesgo que considera la interacción entre los niveles de riesgo y los factores de protección individuales y sociales. Además, alertan del hecho de que esta interacción genera variados niveles de impacto y que la clasificación de una situación como una situación de riesgo, variará de acuerdo con el nivel de desarrollo individual y con el contexto socio-cultural en que esto individuo está inserido.

Reflexionando sobre los principales recursos y temas a considerar para la promoción de la resiliéncia entre jóvenes, en el artículo que estamos revisando, Olsson et al. ${ }^{25}$ estructuran una 
propuesta que se fundamenta en tres niveles de intervención: 1. Nivel individual - Resiliéncia constitucional (características biológicas); Sociabilidad; Inteligencia y Atributos personales. 2. Nivel familiar - básicamente la ayuda y el apoyo familiar. 3. Nivel del ambiente social - el status socio-económico; la experiencia escolar y el soporte de la comunidad.

Para Gardner ${ }^{26}$, la intervención articulada de los tres niveles señalados anteriormente pueden ser en gran medida encontrados en los proyectos de intervención social que deben contar con un cuadro de educadores que incorporen en ellos mismos el conocimiento que se espera que impartan. El autor advierte que a menos que los educadores estén familiarizados y sientan cierta sensación de propiedad en relación al objetivo, método y propuesta socioeducativa del proyecto, todo el esfuerzo educativo estará llamado a fracasar.

En este caso, el papel del educador social se ubica en el campo de ayudas y apoyos que los programas educativos de intervención psicosocial ofrecen a la comunidad. En la medida que dichos programas incorporan la idea de movilizar al mayor número de colectivos y segmentos de la población, se impone la participación de todos los llamados en la tarea de repensar las metas, los objetivos y las actitudes. Consideramos al educador social como fuente de resiliéncia, cuando lidera desde el lugar que ocupe la coordinación de acciones que fomentan la ayuda y el apoyo al trabajo biográfico de los sujetos jóvenes en las favelas. Por poner un ejemplo, un campo de acciones a promover tiene que ver con el compañerismo y la amistad entre iguales ${ }^{26}$.

Avanzando en la comprensión del efecto del grupo social en la promoción de factores resilientes, cabe presentar el estudio sobre interacción social y abandono escolar en favelas de Rio de Janeiro publicado por Vasconcellos \& Rocha $^{27}$ en que se concluye que los amigos ejercen un efecto en el comportamiento de los jóvenes más importante que el efecto producido por la familia y recomiendan que las políticas públicas para los jóvenes pasen a actuar con los pares. En el mismo sentido, Oliva ${ }^{28}$ recuerda que existe suficiente apoyo empírico a la importancia que tienen las relaciones interpersonales para el desarrollo humano del joven, especialmente para el desarrollo socio-moral, el desarrollo de la empatía y de la habilidad de adopción de perspectivas. Por lo tanto, la estimulación proveniente de la oportunidad de vivir en un ambiente apropiado en el periodo de la juventud es esencial para que el ce- rebro desarrolle todo su potencial como parte de un dinámico sistema de esfuerzo del organismo para mantener su existencia por medio del equilibrio frente a los múltiples y incesantes factores que promueve el medio socio-cultural en que él está inserido.

En un análisis crítico del concepto de favela, destacamos la dualidad contradictoria en la forma de percibir el fenómeno y a la ambigüedad de los rasgos que se le atribuyen, tanto si se mira el fenómeno desde el lado oscuro de la inseguridad ciudadana, con todo lo que ello comporta, como desde su cara más luminosa proyectada desde las raíces culturales cariocas y desde los actuales esfuerzos por conseguir los mismos derechos y oportunidades para todos. En consecuencia, la presencia de riesgo, su intensidad y su contenido puede variar de una favela a otra y afectar de forma diferencial a las familias y los jóvenes de una misma favela.

En este contexto de busca por la igualdad de oportunidades situamos el fenómeno de la adaptación positiva o resiliéncia ${ }^{29}$ de los jóvenes que, a pesar de vivir bajo condiciones de riesgo, apuntan hacia una otra vida. La reformulación de la intervención social desde el marco conceptual de la resiliéncia ha supuesto incorporar las revisiones sobre las investigaciones que relacionan la resiliéncia con la buena adaptación y los buenos logros en contextos de riesgo o en situaciones calificadas como adversas. Masten ${ }^{30}$ ha recopilado las evidencias disponibles y confirmadas una y otra vez sobre el papel protector de la resiliéncia.

De acuerdo con la categorización de Garmezy $^{31}$, las tres principales macrocategorías en que pueden agruparse los factores protectores (atributos de la persona; calidad de la vida en familia; y sistemas de apoyo externo a las relaciones familiares), el estudio de los atributos (buena inteligencia, temperamento positivo y actitud positiva hacia sí mismo) correlaciona con la superación exitosa de las dificultades bajo condiciones de bajo riesgo y alto riesgo.

En este sentido, Luttar y Cicchetti ${ }^{32}$ interpretan que las cualidades individuales de la persona pueden representar la fuente de donde emana el potencial que el individuo necesita para contrabalancear la adversidad con los propios activos que deben superan la carga de riesgo. Con la convergencia de resultados sobre la interacción entre factores (protectores y de riesgo) la resiliéncia adquiere un significado más complejo. Y además incorporar la interacción de la persona con el medio. La individualidad psicobiológica (atributos individuales) forma parte del nicho ecológico 
de las relaciones en expansión, implicando diferentes ordenes de sistemas. Dada la importancia que tiene para los sistemas de adaptación humana el desarrollo de competencias bajo condiciones favorables o desfavorables, el empuje resiliente ha de emerger de la actividad ordinaria de vivir en la familia, la escuela, el trabajo, o participar de proyectos educativos ${ }^{32,33}$.

En sintesis, a partir de un trabajo de Masten ${ }^{30}$ los principales factores predictores de resiliéncia son: Uno o más padres efectivos; Cuidados de otros adultos; Habilidades cognitivas, de atención y de resolución de problemas; Reguladores efectivos de emociones y atención; Autoestima y autoeficacia percibida como positivas; Esperanza; Afiliaciones religiosas; Aptitudes valoradas por la sociedad; Amistades adaptadas; Ventajas socioeconómicas; Escolarización efectiva; Comunidad o contexto favorecedor (centros sociales, escuelas, etc.). Por último, siguiendo las consideraciones que hacen Masten y Powell ${ }^{34}$, las políticas y las prácticas de intervención deben centrarse más en mejorar las competencias y en el fortalecimiento de la experiencia de protección que la persona va acumulando en la vida.

\section{El Rol de los Programas Educativos en el Cambio de Escenario}

Jóvenes que pasan el tiempo en comunidades que son ricas en oportunidades educativas experimentan menos riesgo y muestran pruebas de un desarrollo mas positivo. Los programas educativos promueven nuevas oportunidades de desarrollo que se revela en un desarrollo físico e intelectual. Los programas educativos promueven una sinergia entre familia, escuela, y comunidad ampliando la acción de los factores de resiliéncia y protección social ${ }^{2,23,24}$.

En Brasil, muy pocos programas integrados han recibido la clase de completo evaluación experimental necesaria para se hacer una recomendación firme de reproducir el programa en su totalidad. Sin embargo, hay pruebas suficientes de una variedad de fuentes para hacer recomendaciones sobre algunos principios para la promoción del desarrollo como el ejemplo del clásico programa Cultura Viva em tempos de Gilberto Gil como Ministro da Cultura do governo Lula.

Ya en el panorama internacional, el National Research Council and Institute of Medicine en Estados Unidos de America (NRCIM/USA) ${ }^{35}$ propone algunas directrices para los programas educativos que tienen como objetivo promover el desarrollo positivo de la juventud. De acuerdo con los investigadores, hay que considerar dos panoramas posibles: Una parte de los jóvenes, a despecho de las privaciones, lo hacen muy bien y consiguen alcanzar un nivel positivo de desarrollo humano; otra parte de los jóvenes, en los contextos de desventaja y exclusión social, asumen riesgos peligrosos y lo hacen mal.

Para el Instituto Norteamericano, los programas educativos amplían las oportunidades de los jóvenes, sin embargo, no garantizan su desarrollo positivo al corto plazo. En las conclusiones del informe, los autores afirman que más allá de la eliminación de los problemas, uno necesita de habilidades, conocimiento y variedad de otros activos personales y sociales para funcionar bien durante la transición de la adolescencia para la edad adulta. Así una vista más amplia, y más integrada de la ayuda necesaria a la juventud desfavorecida para realizar su potencial lleno gana el crédito más amplio en el mundo de la política y también en la educación ${ }^{35}$.

En las conclusiones del informe que realiza un análisis de dos importantes programas educativos basados en la actividad artística grupal, el proyecto Spectrum de la Universidad de Stanford y el proyecto Zero de la Universidad de Harvard, Gardner $^{26}$ afirma que los proyectos educativos, desde una perspectiva comprensiva del proceso de desarrollo humano, necesitan en cierto modo considerar el espectro completo de las capacidades y los talentos mostrados por los seres humanos maduros en diversas culturas. El investigador afirma aún que en realidad se podría decir que el individuo más especializado en una sociedad ha integrado de un modo positivo estas diversas formas de conocimiento en el interior de la envoltura de una disciplina u oficio culturalmente valorado, aunque para el desarrollo de los jóvenes tal nivel de especialización y especificidad no sea recomendable ${ }^{26}$.

Cuando los jóvenes se encuentran involucrados en programas educativos ricos y atrayentes que invocan una variedad de modos de representación, cuando cuentan con la oportunidad para interactuar y comunicarse con individuos en la misma situación de aprendizaje con dialogo horizontal y propuestas curriculares integradas, muy raramente dejan de aprovechar la oportunidad para su desarrollo humano. La experiencia en el análisis de los proyectos permite al investigador afirmar que Cuando los jóvenes se involucran de forma positiva con la propuesta de un programa educativo ellos frecuentemente relatan una ampliación de sus potencialidades y el desarrollo de nuevas capacidades 
impulsionados por la participación en un proyecto que amplia sus oportunidades de desarrollo personal $^{26}$.

Los jóvenes que comparten de la misma manera la vulnerabilidad del ambiente, tienen juntos, por medio del programa de intervención social, el desafío de desarrollar un sistema de inteligencia que les permita vivir el presente y crecer de forma positiva o satisfactoria. Desde un punto de vista educativo, la estimulación de un crecimiento positivo con jóvenes vulnerables o vulnerados pasa por identificar la presencia de factores protectores de la capacidad de inserción social, entre los cuales la sociabilidad en la vida de familia y la escuela son puntos de anclaje para la formación de redes de relaciones de participación socioafectiva. Pero el crecimiento social de los jóvenes fomentado por las actividades socioeducativas, culturales o informales, reclama, al mismo tiempo, la adquisición de medios económicos a través de la formación das habilidades que son necesarios para llevar a término su proyecto de vida, o sea, desarrollar sus talentos.

Desde nuestra perspectiva, las ideas de Paulo Freire y Carl Rogers son un importante referencial por la insistencia dada por los dos autores a la importancia de la calidad en las relaciones interpersonales y el potencial de desarrollo positivo que los jóvenes pueden vivenciar en los programas educativos. De acuerdo con Freire ${ }^{36}$, para que los educandos desarrollen su autonomía, el educador debe respetar su visión de mundo, lo que significa, tomá-la como punto de partida para la comprensión del rol de la curiosidad como uno de los impulsos primarios de la producción del conocimiento. Para el educador brasileño, enseñar y aprender está relacionado tanto con el esfuerzo metódicamente crítico del educador en desvelar la comprensión de la realidad, así como con el empeño igualmente crítico del alumno en posicionarse como sujeto activo en el proceso de aprendizaje.

Tiene fundamental importancia que el educador perciba la importancia de la escucha de las necesidades del grupo de aprendices lo que exige además de capacidades técnicas y científicas, que el educador posea algunas calidades y virtudes como el amor, la afectividad, el respeto por los otros, la tolerancia, la humildad, la alegría, el gusto por la vida, la apertura al nuevo, aceptar la disponibilidad a los cambios, la esperanza y el dialogo abierto puesto que no es posible la educación transformadora sin el desarrollo de estas calidades de la comunicación fundada en la ver$\mathrm{dad}^{36,37}$.
Esto puesto, es perceptible que el proceso educativo exige una atención al modo como los jóvenes activamente, y con autonomía, construyen las categorías de significado. La producción del significado está fuertemente relacionada a las inversiones emocionales y a la producción del placer que son factores mutuamente constitutivos de quien son los jóvenes, de la opinión que tienen de si mismos y de como ellos construyen una versión particular de su historia de vida y de su futuro. Para Rogers ${ }^{38}$, para que el educando tenga libertad para aprender, el educador tiene que convertirse en un facilitador. Este termino, en verdad, traduce la idea de un educador que establezca un ambiente positivo construido por la confianza en relación al grupo y a las personas. De acuerdo con el autor, el educador necesita aceptar también a si mismo, conocer a si mismo y principalmente, ser él mismo, sin mentiras ni máscaras, lo que es una condición fundamental para cualquiera relación verdadera. Es preciso aún establecer una comprensión empática con los jóvenes de modo que les lleve a comprender el modo como el otro siente los acontecimientos, como expresa sus ideas y también como comunica sus sentimientos.

Así que, el educador, empático, crítico y acogedor no se convierte en dueño de la identidad del alumno, pero también, no pierde la suya. La visión de mundo y los significados colectivos son creados en la subjetividad de cada individuo con base en sus percepciones, estímulos y experiencias, pero también en las intersubjetividades que establece con sus relaciones, familiares, laborales o sociales. Permitir que el alumno crie su propia visão de mundo basado en sus ideas, las experiencias compartidas con otros y el proceso de desarrollo orientado por los educadores ${ }^{38}$.

Podemos considerar que la resiliéncia y la protección social son dos factores fundamentales para el desarrollo positivo de los jóvenes que viven en contextos de vulnerabilidad social. Hay que considerar relevante la observación propuesta por Figueiredo ${ }^{24}$ cuando en un estudio que hace un análisis del proceso de desarrollo humano en contextos de exclusión social y violencia afirma que durante el período en que se implementa un proyecto de intervención social con estrategias promotoras de interacción grupal, los jóvenes frecuentemente desarrollan vínculos positivos con los educadores sociales y, al encontrar protección y estímulo positivo en personas adultas que les proporcionan cuidado y afecto, ellos perciben sus potencialidades y descubren nuevos talentos. 


\section{Consideraciones Finales}

Aún que el contexto social de las favelas sea marcado por la pobreza, la violencia y la exclusión social, también existen importantes factores en la favela que ofrecen oportunidades positivas de desarrollo y, sin duda, la implementación de proyectos de intervención social se constituye en aspecto resiliente fundamental para el cambio del panorama negativo que frecuentemente se asocia a las favelas. Desde esta perspectiva, estamos de acuerdo con Sen ${ }^{5}$ cuando afirma que la función del desarrollo humano es la creación de oportunidades sociales hace una contribución directa a la expansión de capacidades humanas y la calidad de vida.

Esto solo fue posible gracias a Vygotsky ${ }^{39}$ cuando nos permite ampliar la visión del proceso de desarrollo humano cuando afirma que el desarrollo se caracteriza, ante todo, por la producción de cambios dinámicos de carácter orgánico y cultural. En esta perspectiva, el desarrollo superpone la cultura a los procesos de crecimiento, maduración y desarrollo orgánico formando un proceso único.

Así que, el paso de la biología (vivir la vida) a la biografía (responsabilizarse de la vida que se vive) tiene como nudo dramático el desarrollo de la inteligencia y de la personalidad como identidad personal y social. Pero también con la política social encarnada en la preocupación institucional por el futuro de los jóvenes.

Por todo lo expuesto podemos concluir que $e l$ reconocimiento social proporcionado por el vinculo de confianza establecido en programas educativos haz con que los jóvenes se esfuercen por desarrollar cambios de actitudes y empezar a planificar una posibilidad de vida sin violencia. Al tener la oportunidad de establecer lazos sociales positivos con educadores y también con otros jóvenes que comparten de problemas similares, hay un importante cambio de visión de mundo y surge el firme deseo de modificar sus condiciones de vida, sus planteamientos de futuro y aprovechar las nuevas oportunidades.

Aún que el contexto social de las favelas sea marcado por la pobreza, la violencia y la exclusión social, también hay en las favelas diversas oportunidades positivas para el desarrollo humano y, el trabajo de los programas educativos y culturales se constituye en aspecto fundamental de resiliéncia para el cambio del panorama negativo que frecuentemente se asocia a las favelas. La vulnerabilidad social es inversamente proporcional a las oportunidades para el desarrollo, pero, como en una ampolleta de arena, hay un tiempo, un camino. Son diversas variables influyendo en la fuerza resultante, que será el talento de joven para... Cada grano de arena está en diferente punto entre los dos extremos de la ampolleta.

\section{Agradecimientos}

A los jóvenes de las favelas por su lucha cotidiana. al soporte para la investigación aportado por la Agencia Española de Cooperación Internacional y Desarrollo (AECID). Muchas gracias a los profesores e interlocutores en la Universitat Autonoma de Barcelona: Adolf Perinat, Albert Bonillo, Conrad Rodriguez, Santiago Estaún y Maria Tereza Anguera. Y también a todos los educadores sociales que han contribuido con la reflexión sobre las oportunidades de desarrollo positivo para los jóvenes que son generadas a partir de las práticas educativas que se realiza en las favelas. Por fin, gracias a los colegas de trabajo en el NUTES/ UFRJ y en la ENSP/Fiocruz por sus contribuiciones al desarrollo deste estudio. 


\section{Referencias}

1. Instituto Brasileiro de Geografia e Estatística (IBGE). Censo Populacional do ano de 2007. Rio de Janeiro: IBGE; 2008.

2. Figueiredo GO. Promoción del Desarrollo Humano con Jovenes en Favelas de Rio de Janeiro/ Brasil: Vulnerabilidad, Resiliencia e Intervención Social [tese] Barcelona: Universidad Autónoma de Barcelona; 2011.

3. Arpini D. Violencia e Exclusão adolescência em grupos populares. São Paulo: EDUSC; 2003.

4. Perona N, Rocchi G. Vulnerabilidad y Exclusión Social. Una propuesta metodológica para el estúdio de las condiciones de vida. Revista de Temas Sociales 2001; 8:1-9.

5. Sen A. Desarrollo como Liberdade. Barcelona: Editorial Planeta; 2000.

6. Silver H. Social Exclusion and Social Solidarity: Three Paradigms. International Labour Review 1994; 133(56):531-578.

7. Martinez A. Microcrédito y Pobreza-Proyecto de Desarrollo de Comunidades Rurales Pobres [tese]. Málaga: Universidad de Málaga; 2004.

8. Comisión Europea. Programa Comunitario de Acción para Combatir la Exclusión Social 2002-2006. Bruselas: Fondo Social Europeo; 2010.

9. Alkire S. Dimensions of Human Development: Oxford poverty \& Human Development Initiative. World Development 2002; 30(2):181-205.

10. Lenoir R. Les Exclus - Un Francais sur Dix. Paris: Editions du Seuil; 1974.

11. Figueiredo JB, De Haan A. Social Exclusion: in an ILO Perspective. Geneve: International Labour Organization; 1998.

12. Abrahamson P. Exclusión social en Europa: ¿vino viejo en odres nuevos? In: Moreno L, editor. Unión Europea y Estado del Bienestar. Madrid: CSIC; 1997. p.177-141.

13. United Nations (UN). El concepto de exclusion en politica social. Seminario Luchar contra la Exclusión Social. Copenhague: United Nations Press; 1993.

14. Atkinson AB. Social Exclusion, Poverty and Unemployment. In: Hills J, editor. Exclusion, Employment and Opportunity. London: London School of Economics and Political Science; 1998. p.1-20.

15. Brasil. Ministério da Saúde (MS). Sistema de Informações sobre Mortalidade (SIM). Informações de saúde e estatísticas vitais. Brasília: MS/SVS/DASIS; 2004.

16. Arendt H. Sobre a Violência. Rio de Janeiro: Relume Dumará; 1994.

17. Minayo MC. Violência e Saúde. Rio de Janeiro: Editora Fiocruz; 2006.

18. Turcheti N. Public policy for the favelas in Rio de Janeiro: the problem (in) framing [tese]. The Hague: ISS; 2010.

19. Freire P. Pedagogia do Oprimido. $36^{\mathrm{a}}$ ed. Rio de Janeiro: Paz e Terra; 1970.

20. Morin E. A cabeça bem feita: repensar a reforma, reformar o pensamento. $6^{\text {a }}$ ed. Rio de Janeiro: Bertrand Brasil; 2002.

21. Castel R. As Metamorfoses da Questão Social. Petrópolis: Editora Vozes; 1995.

22. Dowdney L. Crianças do tráfico: um estudo de caso de crianças em violência armada organizada no Rio de Janeiro. Rio de Janeiro: 7 letras; 2000.

23. Figueiredo GO, Wimmer GF. Ação Coletiva para a Qualidade de Vida. Cien Saude Colet 2006; 11(1):145154 .
24. Figueiredo GO. Desarrollo Humano en Contextos de Exclusión Social y Violencia: Los Jóvenes en Favelas de Río de Janeiro/Brasil [dissertação]. Barcelona: Universidad Autónoma de Barcelona; 2008.

25. Olsson CA, Bond L, Burns JM, Vella-Brodrick DA, Sawyer SM. Adolescent resilience: a concept analysis. J Adolesc 2003; 26(1):1-11.

26. Gardner H. Educación Artística y Desarrollo Humano. Barcelona: Paidós educador; 1994.

27. Vasconcellos P, Rocha LF. Interação social e evasão escolar nas favelas do Rio de Janeiro - um problema de identificação. Rio de Janeiro: UFRJ; 2006.

28. Oliva A. La adolescencia como riesgo y oportunidad. Infancia y Aprendizaje 2004; 27(1):115-122.

29. Luthar S. Resilience and Vulnerability. Adaptation in the Context of Childhood Adversities. Cambridge: Cambridge University; 2008.

30. Masten AS. Ordinary Magic: Resilience processes in development. Am Psychol 2001; 57(3):227-238.

31. Garmezy N. Stress-resistant children: The search for protective factors. In: Stevenson JE, editor. Recent research in developmental psychopathology. Journal of Child Psychology and Psychiatry 1985; 4:213-233.

32. Luthar S, Cicchetti D. The construct of resilience: Implications for interventions and social policies. Dev Psychopathol 2000; 12(4):857-885.

33. Carretero R. Resiliencia. Una visión positiva para la prevención y la intervención desde los servicios sociales. Nómadas. Revista crítica de Ciencias Sociales 2010; 27(3):91-103.

34. Masten SA, Powell LJ. Resilience Framework for Research, Policy, and Practice. In: Luthar S, editor. Resilience and Vulnerability. Adaptation in the Context of Childhood Adversities. Cambridge: Cambridge University; 2008. p. 1-25.

35. National Research Council and Institute of Medicine (NRCIM). Community Programs to Promote Youth Development. Division of Behavioral and Social Sciences and Education. Washington: National Academy Press; 2002.

36. Freire P. Pedagogía da Autonomia - Saberes Necessários à Prática Educativa. 14a ed. Rio de Janeiro: Paz e Terra; 1997.

37. Freire P. Conscientização, Teoría e Práctica da Libertação: Uma Introdução ao Pensamento de Paulo Freire. 3a ed. São Paulo: Centauro; 1980.

38. Rogers C. Liberdade para Aprender. São Paulo: Martins Fontes; 1983.

39. Vygotsky LS. Historia del desarrollo de las funciones psíquicas superiores. Barcelona: Critica; 1931.
Artigo apresentado em 02/03/2015

Aprovado em 27/10/2015

Versão final apresentada em 29/10/2015 